\title{
Applying the Fluctuation-Dissipation Theorem to a Two-Layer Model of Quasigeostrophic Turbulence
}

\author{
NiCHOLAS J. LUTSKO \\ Program in Atmospheric and Oceanic Sciences, Princeton University, Princeton, New Jersey \\ ISAAC M. HELD \\ NOAA/Geophysical Fluid Dynamics Laboratory, Princeton, New Jersey \\ PABLO ZURITA-GOTOR \\ Universidad Complutense de Madrid, and Instituto de Geociencias, Madrid, Spain
}

(Manuscript received 9 December 2014, in final form 5 March 2015)

\begin{abstract}
The fluctuation-dissipation theorem (FDT) provides a means of calculating the response of a dynamical system to a small force by constructing a linear operator that depends only on data from the internal variability of the unperturbed system. Here the FDT is used to estimate the response of a two-layer quasigeostrophic model to two zonally symmetric torques, both barotropic, with the same sign of the forcing in the two layers, and baroclinic, with opposite sign forcing in the two layers. The supercriticality of the model is also varied to test how the FDT fares, as this parameter is varied. To perform the FDT calculations the data are decomposed onto empirical orthogonal functions (EOFs) and only those EOFs that are well resolved are retained in the FDT calculations. In the barotropic case good qualitative estimates are obtained for all values of the supercriticality, though the FDT consistently overestimates the response, perhaps because of significant non-Gaussian behavior present in the model. Nevertheless, this adds to the evidence that the annular-mode time scale plays an important role in determining the response of the midlatitudes to small perturbations. The baroclinic case is more challenging for the FDT. However, by constructing different bases with which to calculate the EOFs, it is shown that the issue in this case is that the baroclinic variability is poorly sampled, not that the FDT fails. The strategies developed in order to generate these estimates may be applicable to situations in which the FDT is applied to larger systems.
\end{abstract}

\section{Introduction}

There is interest in using the fluctuation-dissipation theorem (FDT) to calculate linear response operators for climate models or, more ambitiously, for the climate system itself. Heuristically, the FDT says that the fluctuations of a system in equilibrium can be used to predict how that system will respond to a small perturbation. Perturb a system of the form

$$
\frac{d \mathbf{x}_{0}}{d t}=M\left(\mathbf{x}_{0}, t\right)
$$

Corresponding author address: Nicholas Lutsko, Program in Atmospheric and Oceanic Sciences, Princeton University, 300 Forrestal Rd., Princeton, NJ 08540.

E-mail: lutsko@princeton.edu by a small force

$$
\frac{d \mathbf{x}_{f}}{d t}=M\left(\mathbf{x}_{f}, t\right)+\Delta \mathbf{f}
$$

(we restrict all of our considerations to the case of a timeindependent force). Here $\mathbf{x}_{0}$ is the mean state vector of the undisturbed system and $\mathbf{x}_{f}$ is the mean state vector of the forced system. The FDT says that the fluctuations of the unperturbed system can be used to calculate a linear response operator, $\mathbf{L}$, with which one can directly compute the response of the time-averaged solution

$$
\left\langle\mathbf{x}_{f}-\mathbf{x}_{0}\right\rangle=\delta \mathbf{x}=\mathbf{L} \Delta \mathbf{f} .
$$

Much effort has been devoted to understanding the internal variability, or "fluctuations," in observations and 
in models, so it is intriguing to be able to use this framework to predict how these systems respond to perturbations.

It is perhaps unsurprising then that the FDT has a relatively long history in the climate science literature, beginning with the seminal work of Leith (1975), followed among others by Bell (1980), Majda et al. (2005), Gritsun and Branstator (2007), Ring and Plumb (2008), and Majda et al. (2010). Recently, the assumptions underlying the FDT and its applicability for the climate system have been addressed (Majda et al. 2005; Gritsun and Branstator 2007) and we will be focusing here on the "quasi Gaussian" formulation of the FDT:

$$
\mathbf{L}=\int_{0}^{\infty} \mathbf{C}(\tau) \mathbf{C}(0)^{-1} d \tau
$$

where $\mathbf{C}$ represents covariance matrices and $\tau$ represents time lags. While nonparametric versions that do not assume multivariate Guassian statistics have been described in the literature (Cooper and Haynes 2011), the practical implementation of more general versions appears to be very formidable. Other options, such as the blendedresponse algorithm of (Abramov and Majda 2009) require information about the underlying dynamical operator. Our interest here is in the version that requires only the covariance matrix in an equilibrium state, with no knowledge required of the underlying dynamics that produce these covariance statistics. We refer to this classical formulation as simply the FDT in the following.

The accuracy of the FDT in climate problems of interest remains unclear, however. Gritsun and Branstator (2007) found good agreement between their FDT estimates and the response of an atmosphere-only general circulation model (GCM) to heating perturbations; we view this as the most promising result to date. Ring and Plumb (2008) were not able to obtain accurate estimates for the response of a dry dynamical core to various zonally symmetric torques and thermal forcings using a principal oscillation pattern (POP) analysis, rather than the full FDT. Whether the full FDT would work better in this case is unclear. Previous work has also focused on more idealized systems, such as the Lorenz-96 model and variants of Burger's equation (see, e.g., Majda et al. 2005), in order to develop the theoretical framework of the FDT and to compare alternative formulations. We believe that there is room for work on intermediatecomplexity systems with large numbers of degrees of freedom, motivated by climate change issues, but in which the covariance statistics are more easily generated than for primitive equation models on the sphere. These intermediate systems can also allow for more careful study of how best to choose a basis on which to project the evolution of the model state and how best to truncate this basis so that it is small enough to allow robust estimation of the covariance matrix. The goal is to avoid subjective choices that complicate the testing of the robustness and accuracy of the FDT.

With this motivation, in the following work we have applied the FDT to a turbulent, baroclinically unstable jet in a two-layer quasigeostrophic (QG) model. The two-layer model is simple enough that we can relatively quickly gather a large amount of data, ensuring robust statistics, and conduct extensive sensitivity tests. At the same time, its simplicity makes it a strict test of the FDT's value to see if it can provide estimates for the model's response to forcings that are qualitatively useful if not fully quantitatively accurate.

Moreover, the two-layer model captures the essential features of atmospheric circulation in the midlatitudes and so is also of much scientific interest. A particular motivation for this work is the presence of annular-mode-like variability in this model. Annular modes are the dominant mode of variability in the zonal-mean winds in the extratropics and a clear relationship has been seen in idealized models between the decorrelation time of this mode and the response of the midlatitude circulation to forcing (Chan and Plumb 2009; Gerber et al. 2008; Butler et al. 2010). This suggests that there is an FDT-like connection between the autocorrelation of the annular modes and the response of the midlatitude circulation to forcing, though previous applications of the FDT have not been quantitatively successful (Ring and Plumb 2008; F. C. Cooper and P. H. Haynes 2013, personal communication). If the FDT does hold, it would be concerning as many GCMs have overly persistent annular modes, potentially leading to overestimates of the actual response of midlatitudes to small perturbations, with consequences for the response of midlatitude circulation to the ozone hole as one important example.

Finally, several papers have investigated the complex structure of the autocorrelation of the annular modes (Lorenz and Hartmann 2001; Gerber et al. 2008; ZuritaGotor et al. 2014; Zurita-Gotor 2014). In particular, Zurita-Gotor et al. (2014) showed that this autocorrelation includes high- and low-frequency components that reflect the effects of positive and negative eddy feedbacks. This may explain the lack of quantitative agreement in Ring and Plumb (2008), as simplified methods that effectively assume the structure of the correlations are unlikely to give accurate estimates and also justifies our use of the full FDT, which integrates over different time scales.

The rest of this report is structured as follows. Our methodology is presented in the next section followed in section 3 by an examination of how the model responds 
to small perturbations and how its behavior varies as the supercriticality of the control climate's jet is varied. The results of the FDT calculations are then presented in section 4 before we end with our conclusions.

\section{Methodology}

\section{a. Model formulation}

A nondimensional version of the dynamical equations of our QG model is given by

$$
\begin{aligned}
\frac{\partial q_{k}}{\partial t}+J\left(\psi_{k}, q_{k}\right)= & -\frac{1}{\tau_{d}}(-1)^{k}\left(\psi_{1}-\psi_{2}-\psi_{R}\right) \\
& -\frac{1}{\tau_{f}} \delta_{k 2} \nabla^{2} \psi_{k}-\nu \nabla^{4} q_{k},
\end{aligned}
$$

where $q_{k}=\nabla^{2} \psi_{k}+(-1)^{k}\left(\psi_{1}-\psi_{2}\right)+\beta y$ is the potential vorticity in the upper $(k=1)$ and lower $(k=2)$ layers, $\psi_{k}$ represents the corresponding streamfunctions, $\tau_{d}$ is a Newtonian relaxation time scale, $\tau_{f}$ is a Rayleigh friction time scale acting only in the lower layer (this is indicated by the Kronecker $\delta$ function), and $\nu$ is a hyperdiffusion coefficient.

The model is set up as a zonally periodic channel with a baroclinic zone in the center of the domain creating a baroclinically unstable jet. This jet was obtained by setting the radiative equilibrium temperature $\left(\psi_{1}-\psi_{2}\right)$ profile to a hyperbolic secant centered at $y=0$. In radiative equilibrium (i.e., in the absence of eddy fluxes) the lower-layer flow $\psi_{2}$ is identically zero so that the zonal flow in the upper layer $U_{1}(y)=-\partial \psi_{1} / \partial y=-\partial \psi_{R} / \partial y$ is

$$
-\frac{\partial \psi_{R}}{\partial y}=\operatorname{sech}^{2}(y / \sigma)
$$

The values of the model parameters are nondimensionalized versions of those used by Zurita-Gotor et al. (2014), with the velocity scale set by the strength of the baroclinically unstable jet and the length scale set by the radius of deformation. With these parameters the jet width $\sigma$ is $3.5, \beta$ is $0.196, \tau_{f}$ is $\sim 15, \tau_{d}$ is $\sim 100$, and $\nu$ is 0.01 . We choose to follow Zurita-Gotor et al. (2014) in the choice of parameters because they result in qualitatively realistic annular-mode variability. With this nondimensionalization, Phillips's stability criterion for uniform vertical shear in the absence of dissipation is $\beta<1$. The number of model "days" (time units) making up 1 Earth day is roughly 5 . The code is spectral and so sponges are required near the northern and southern boundaries; these take the form of strong exponential damping with the same half-width as the jet. The domain is wide enough that the sponges do not affect the dynamics, as we have verified. The nondimensionalized zonal and meridional widths are $L_{x}=46$ and $L_{y}=68$,
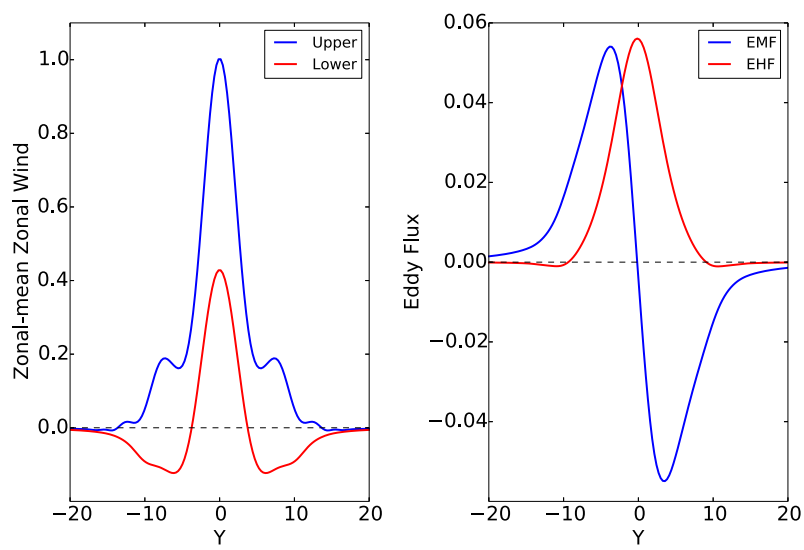

FIG. 1. (left) Time-averaged zonal-mean zonal wind profiles for both layers from a $10^{5}$-day integration of the two-layer model using the parameter settings of Zurita-Gotor et al. (2014) and (right) the time-averaged zonal-mean EMF and EHF.

while 42 and 85 zonal and meridional Fourier modes are retained, respectively, using a standard spectral transform algorithm that computes the projection of nonlinear products onto the retained modes exactly.

Other than differences in the numerical algorithm, the only difference with Zurita-Gotor et al. (2014) is the form of the hyperdiffusion, but the magnitude of the hyperdiffusion has a very small effect on the model climates. The time-averaged zonal-mean zonal winds and the time-averaged zonal-mean eddy heat and momentum fluxes (EHF and EMF, respectively) are shown in Fig. 1.

This model is also similar to that used by DelSole (1996) in a study using POP analysis. DelSole found that the empirical operator obtained by the POP analysis depends on time lag, inconsistent with the Markov model approximation underlying that technique. The FDT does not share this limitation.

\section{b. Control simulations and perturbations}

To estimate the covariance statistics the model was integrated for $10^{6}$ model days, with the first $10^{3}$ model days discarded to ensure that the model had equilibrated. Data were recorded every four model days (i.e., every 0.8 Earth days). Tests indicated that higherfrequency sampling produces no significant change in the results. The resulting dataset was then divided into an ensemble of 10 members, each representing approximately $10^{5}$ model days of data, to estimate the sampling error.

The most general zonally symmetric perturbation in this model can be expressed as zonally symmetric source terms in the upper- and lower-layer potential vorticity equations. For a heating perturbation $Q$ and upper- and lower-layer torques $\left(f_{1}, f_{2}\right)$, we have 


$$
\begin{aligned}
& \frac{\partial \overline{q_{1}}}{\partial t}=\cdots-\frac{\partial f_{1}}{\partial y}+Q \quad \text { and } \\
& \frac{\partial \overline{q_{2}}}{\partial t}=\cdots-\frac{\partial f_{2}}{\partial y}-Q .
\end{aligned}
$$

Equivalently we can think of forcing the barotropic and baroclinic components of the flow (the sum and difference of the upper and lower layers). The response to heating is equivalent to the response to a purely baroclinic zonal force with $f_{1}=-f_{2}$, and we speak equivalently of the baroclinic case as the response to heating $Q$ or to a baroclinic zonal force. We consider pure barotropic and pure baroclinic forcing. The FDT prediction for a more general perturbation (momentum forcing in the upper layer only, for example) can be obtained from the linear superposition of barotropic and baroclinic special cases.

We describe the baroclinic case first. Given the knowledge that the dominant mode of variability in this model is annular-mode-like (Zurita-Gotor et al. 2014; see also Figs. 3 and 5 below), consisting of a meridional shift in the winds and storm track, just as in the atmosphere, we design the latitudinal structure of the forcing to give the FDT the best chance to generate robust responses. To do this, we choose $Q$ proportional to the derivative of the radiative equilibrium temperature field. For small perturbations, this is equivalent to a latitudinal shift in the baroclinic zone:

$$
Q=Q_{0} \frac{\partial \psi_{R}}{\partial y} .
$$

As described below, when performing the FDT computations we decompose the model output into empirical orthogonal functions (EOFs). To perform this decomposition a basis with which to compute the covariance matrix and a corresponding norm are required. Since the climate statistics of the control and perturbed models are zonally symmetric only the EOFs of the zonal-mean circulation are required by the FDT. Although the numerical model is formulated using potential vorticity as the prognostic variable, better results are obtained when performing the FDT calculations using the zonal-mean zonal wind to define the basis and norm. Using the zonal-mean potential vorticity variance as the norm when computing the EOFs results in EOFs with more finescale structure than if the zonal-mean zonal wind variance is used as the norm. This finescale structure magnifies errors in the FDT calculations and so the results are cleaner when using the zonal-mean zonal wind. To directly apply the FDT formalism in this basis the potential vorticity forcings need to be converted into zonal wind tendencies [just as in Ring and Plumb (2008)]. For our baroclinic forcing the result is

$$
\frac{\partial \bar{u}_{k}}{\partial t} \equiv(-1)^{k} F,
$$

where $k$ indicates the model level and $F(y)$ is obtained by solving

$$
\left(\frac{\partial^{2}}{\partial y^{2}}-2\right) F=\frac{\partial Q}{\partial y}=Q_{0} \frac{\partial^{2} \psi_{R}}{\partial y^{2}}
$$

This zonal wind tendency is shown in Fig. 2b. Physically, it forces a southward shift of the baroclinic component of the radiative equilibrium jet (see Fig. 2a) and the response to this perturbation is shown in Fig. 2c.

The relationship between $Q$ and the zonal tendency $F$ accounts for the effects of the mean meridional circulation. In the barotropic case of equal zonal forces in the two layers there is no induced meridional circulation and the imposed potential vorticity tendency is proportional to the derivative of the zonal force in both layers; that is,

$$
\frac{\partial \overline{u_{k}}}{\partial t}=f_{k},
$$

where $f_{k}$ is as in Eq. (8). We choose the profile of this force to be similar to that obtained from Eq. (11) for the baroclinic zonal wind tendency by simply setting $f_{1}$ and $f_{2}$ to

$$
f_{1}=f_{2}=f_{0} \frac{\partial^{2} \psi_{R}}{\partial y^{2}}
$$

This zonal wind tendency represents a southward shift of the barotropic component of the radiative equilibrium jet and is shown in Fig. 2d. The response is shown in Fig. 2e; note that the barotropic and baroclinic forcings produce qualitatively similar equivalent barotropic responses.

The amplitude of the forcings must be small enough that the responses are in the linear regime and large enough that these linear responses can be isolated accurately from the model's variability. We have found that $f_{0}=Q_{0}=0.01$ is safely in this range, verifying that the changes in the responses are negligible when the forces are increased or decreased by a factor of 2 . Forces of this strength shift the baroclinic zone by roughly $24 \%$ of the half-width of the jet $(\sigma)$. Tests in which the domain size was doubled but the resolution was kept constant indicated no sensitivity of the responses to domain size.

After performing these calculations with the parameters of Zurita-Gotor et al. (2014), the simulations were repeated while varying $\beta$ in increments of 0.05 from the original value of roughly 0.2 up to a value of 0.4 (when $\beta$ is decreased from 0.2 , the width of the domain begins to affect the model's response). This induces substantial 

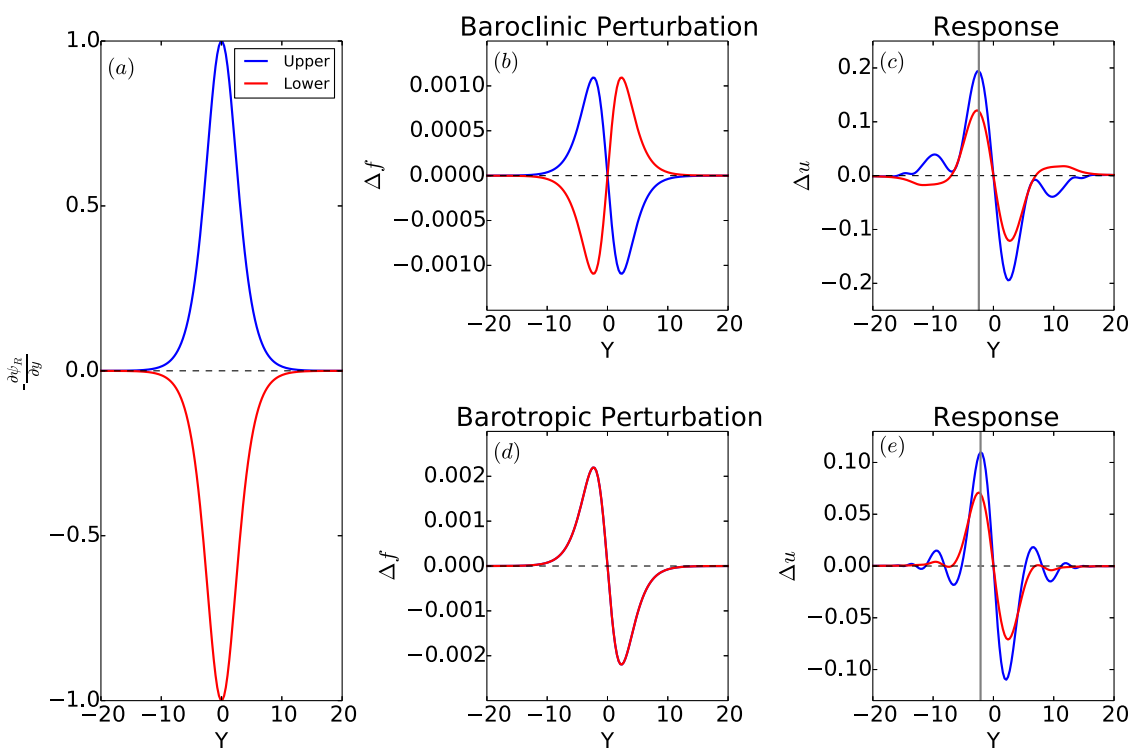

FIG. 2. (a) Profile of $\partial \psi_{R} / \partial y$, (b) the baroclinic perturbation to the zonal momentum equations applied to the two-layer model, and (c) the resulting model response (the zonal wind tendency is inverted from the thermal forcing); (d) the barotropic perturbation (note: the perturbations to the upper and lower layers plot on top of each other) and (e) the model response. The vertical gray lines in (c) and (e) show the position of the max $\Delta u$ metric.

changes in the model behavior, which will be discussed in section 3, and was done in order to test how the FDT fared under different parameter settings. In every case the forces were again halved and doubled to check that the responses are linear functions of the perturbation strength.

\section{c. Regularization strategy}

As has been discussed by, for example, Gritsun and Branstator (2007), errors in the calculation of the linear operator $\mathbf{L}$ are a function of the length of the available data, $n$, and the "effective" number of degrees of freedom (Martynov and Nechepurenko 2004). The former simply arises from the central limit theorem, which says that the errors in the estimates of the linear operator $\mathbf{L}$ will be proportional to $1 / \sqrt{n}$ (Gritsun and Branstator 2007). The second is a result of the $\mathbf{C}(0)^{-1}$ term. For a system with many degrees of freedom, $\mathbf{C}(0)$ is likely to be an ill-conditioned matrix, resulting in large errors when its inverse is estimated. However, only a subset of the total degrees of freedom are relevant for the FDT computation, while the other degrees of freedom simply decay and so cause errors in the estimation of $\mathbf{C}(0)$. The goal of regularization is to minimize the effects of the latter degrees of freedom.

The most commonly used way of regularizing the $\mathbf{C}(0)$ matrix in FDT studies is to truncate the number of included degrees of freedom. This strategy involves a balance between including enough dimensionality to capture the behavior of the data and not including unnecessary dimensionality. To do this we have followed previous works by projecting the data onto the EOFs and then truncating the number of EOFs included in the calculations.

The two-layer model with a climate that is symmetric about the center of the channel has two types of zonalmean zonal wind EOFs: half are symmetric about the center of the channel and the other half are antisymmetric. The leading EOF has an antisymmetric annularmode-type structure, the next leading EOF has a pulsing symmetric structure, and so on. Symmetric perturbations and responses only project onto the symmetric EOFs while antisymmetric perturbations and responses only project onto the antisymmetric EOFs. Hence only the antisymmetric EOFs have been used here as the perturbations described above are both antisymmetric. For some cases the higher ranking EOFs were not purely symmetric or antisymmetric owing to sampling issues, but they were still recognizably symmetric or antisymmetric and so were binned accordingly. Tests indicated that this separation did not affect the results.

In addition to this partition we initially considered two ways of defining the EOFs: calculating them separately for each layer or for both layers simultaneously. We term the former "uncoupled EOFs" and Fig. 3a shows the leading EOF calculated for each layer separately. We term the latter "coupled EOFs" and Fig. $3 \mathrm{~b}$ shows the leading EOF calculated using both layers simultaneously. 

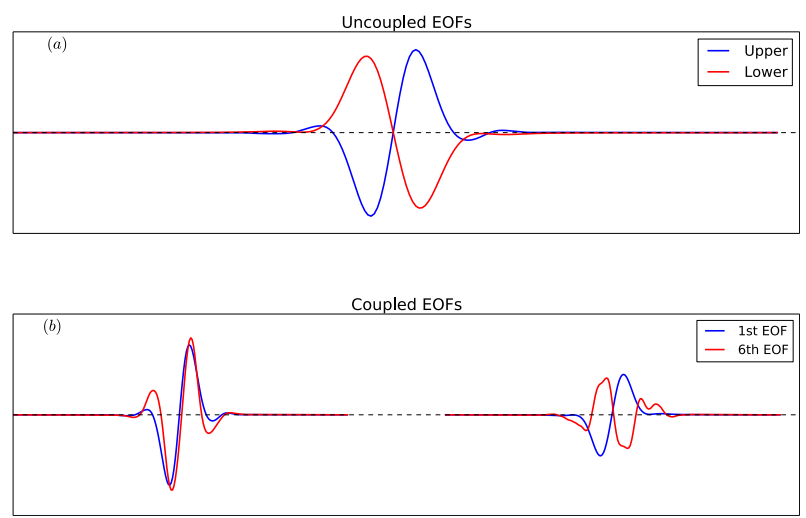

FIG. 3. (a) The leading uncoupled EOF in each layer when $\beta=0.2$; (b) the leading coupled EOF (blue) and the sixth antisymmetric coupled EOF (red) for the same case. The lines to the left correspond to the upper layer and the lines to the right correspond to the lower layer.

The calculations below were carried out with both kinds of antisymmetric EOF but the calculations using the coupled EOFs were expected to have less error than those carried out with the uncoupled EOFs as the lag- 0 covariance matrix of the coupled EOFs is likely to be more well-conditioned than that for the uncoupled EOFs, being diagonal by definition. A physical interpretation for this is the strong coupling between the upper and lower layers through the potential vorticity; hence, treating the layer EOFs as independent degrees of freedom produces a poorly conditioned covariance matrix. We do not show the results of calculations using uncoupled EOFs here, but this was indeed the case.

Having defined the EOFs, the question raised in the introduction of how many EOFs to retain remains. This is a central concern whenever the FDT is applied to large systems and is discussed in detail in section $4 \mathrm{a}$.

\section{d. Testing Gaussianity}

The formulation of the FDT we have used assumes that the system is Gaussian. This is unlikely to be the case in practice, but to our knowledge the relationship between non-Gaussian behavior and bias in FDT estimates is still unclear. We have not attempted to explore this question rigorously but to help address this issue we have used Mardia's test to calculate the multivariate skewness $\gamma$ and kurtosis $K$, and so test for multivariate Gaussianity. These are given by

$$
\begin{aligned}
\gamma & =\frac{1}{n^{2}} \sum_{i=1}^{n} \sum_{j=1}^{n}\left[\left(\mathbf{x}_{i}-\overline{\mathbf{x}}\right)^{\mathrm{T}} \mathbf{C}(0)^{-1}\left(\mathbf{x}_{j}-\overline{\mathbf{x}}\right)\right]^{3} \text { and } \\
K & =\frac{1}{n} \sum_{i=1}^{n}\left[\left(\mathbf{x}_{i}-\overline{\mathbf{x}}\right)^{\mathrm{T}} \mathbf{C}(0)^{-1}\left(\mathbf{x}_{i}-\overline{\mathbf{x}}\right)\right]^{2}
\end{aligned}
$$

where $\mathbf{x}$ is the state vector of length $d$ and $n$ is the number of data points in each ensemble member $\left(10^{5}\right)$ (Wilks 2006). We have calculated $\gamma$ and $K$ for each ensemble member separately and then averaged the results. Overbars denote mean values. With these definitions two test statistics can be defined, $T_{\gamma}$ and $T_{K}$ :

$$
\begin{aligned}
T_{\gamma} & =\frac{n}{6} \gamma \text { and } \\
T_{K} & =\sqrt{\frac{n}{8 d(d+2)}}[K-d(d+2)] .
\end{aligned}
$$

For a multivariate normal system $T_{\gamma}$ will have a $\chi^{2}$ distribution with $[d(d+1)(d+2)] / 6$ degrees of freedom while $T_{K}$ will have a normal distribution with mean zero and standard deviation one (Wilks 2006). Hence, once $T_{\gamma}$ and $T_{K}$ are estimated $p$ values can be calculated to test the assumption of Gaussianity: if the $p$ value of each of these is less than 0.05 the null hypothesis that the data is normally distributed can be rejected at the $95 \%$ level.

\section{e. Another computational note}

Estimating $\int_{0}^{T} \mathbf{C}(\tau) d \tau$ is very time consuming: the most obvious algorithms require $O\left(d^{2} n r\right)$ operations, where $n$ and $d$ are as before and $r$ is the number of data points corresponding to a lag $T$. In an unpublished manuscript, Cooper and Haynes have shown that this can be reduced to an $O\left(n d^{2}\right)$ calculation by a suitable rearrangement of the double summation over the $r$ time lags and $n$ data points. As it has not previously been published the details of this algorithm are given in the appendix.

\section{Model results}

\section{a. Behavior of the model as $\beta$ is increased}

Varying $\beta$ substantially affects the model's behavior, even before perturbations are applied. For instance, the time-averaged behavior of the zonal jets changes noticeably as $\beta$ is increased. In the upper layer the jet, which is similar to the radiative equilibrium jet when $\beta=0.2$, becomes narrower and strengthens, while adding structure in the "wings" (see top panel of Fig. 4). In the lower layer the jet becomes narrower but its strength does not change significantly as $\beta$ is increased (see bottom panel of Fig. 4).

From an FDT perspective the variability of the model is centrally important and this is also affected by increasing $\beta$, with the flow becoming more persistent. This can be seen in Fig. 5, which shows the autocorrelation of the first principal component for the different cases. Clearly, the annular mode becomes more persistent as $\beta$ is increased so that when $\beta=0.4$ the autocorrelation oscillates significantly even at very long lags. This is 

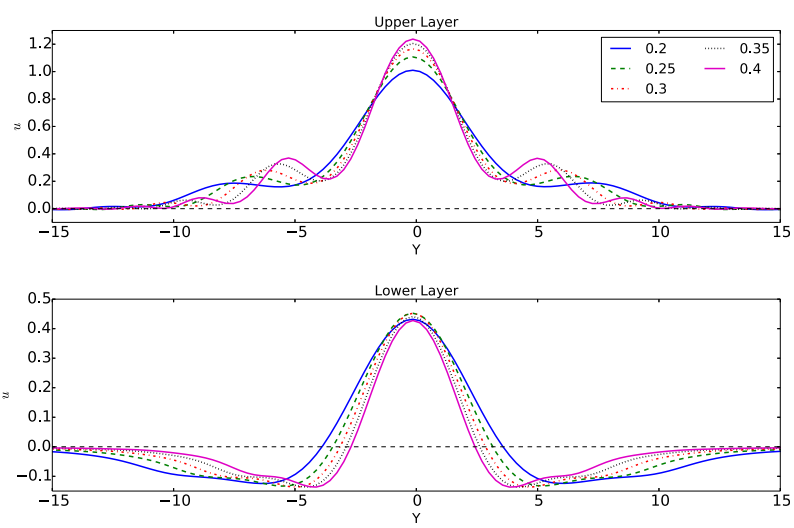

FIG. 4. Comparison of the time-averaged zonal-mean zonal winds as $\beta$ is varied from 0.2 to 0.4 . (top) The upper layer and (bottom) the lower layer.

associated with the development of a low-frequency peak in the eddy momentum forcing spectrum as $\beta$ is increased (not shown). Another noticeable feature, which may be related, is that the transition between the negative eddy feedback, which causes the autocorrelation to decay rapidly, and the positive eddy feedback, which causes the autocorrelation to decay less rapidly, takes place sooner and becomes noticeably sharper as $\beta$ is increased [see inset of Fig. 5; also see Zurita-Gotor et al. (2014) for a discussion of the positive and negative eddy feedbacks].

In addition to becoming more persistent as $\beta$ is increased, the model also becomes less Gaussian. This can be seen in the left panels of Fig. 6, which plot the multivariate skewness and kurtosis test statistics for the first six coupled antisymmetric EOFs for three values of $\beta$ (note: in this and in all subsequent figures the plus or minus one standard deviation envelope is calculated by dividing the standard deviation of the ensemble estimates by $\sqrt{10}$ ). The skewness increases monotonically with $\beta$ and, while the kurtosis does not follow as clear a trend, the $\beta=0.2$ data are closest to the $95 \%$ confidence interval. In all cases the data show significant departures from Gaussianity. This is concerning for the FDT, which of course assumes that the data are at least approximately Gaussian and may be of relevance to the results below.

To test if the non-Gaussianity arises from the joint probability distributions or if the univariate probability distributions for the individual modes are non-Gaussian, we have removed the coupling between modes by randomly disordering each of the principal component time series. This substantially reduces the departures from Gaussianity (see right panels of Fig. 6), though the data are still not quite Gaussian. There is also less dependence on $\beta$. The interactions between modes thus explain much of the departures from Gaussianity as well as the increasingly non-Gaussian behavior as $\beta$ is increased.

\section{b. Model responses}

The model responses to the two perturbations in the initial setting with $\beta=0.2$ are shown in Figs. $2 c$,e. In both cases the response is antisymmetric, has the same sign in each layer, and is stronger in the upper layer than in the lower layer. There is also a significant response in the wings in each case, suggesting that the response

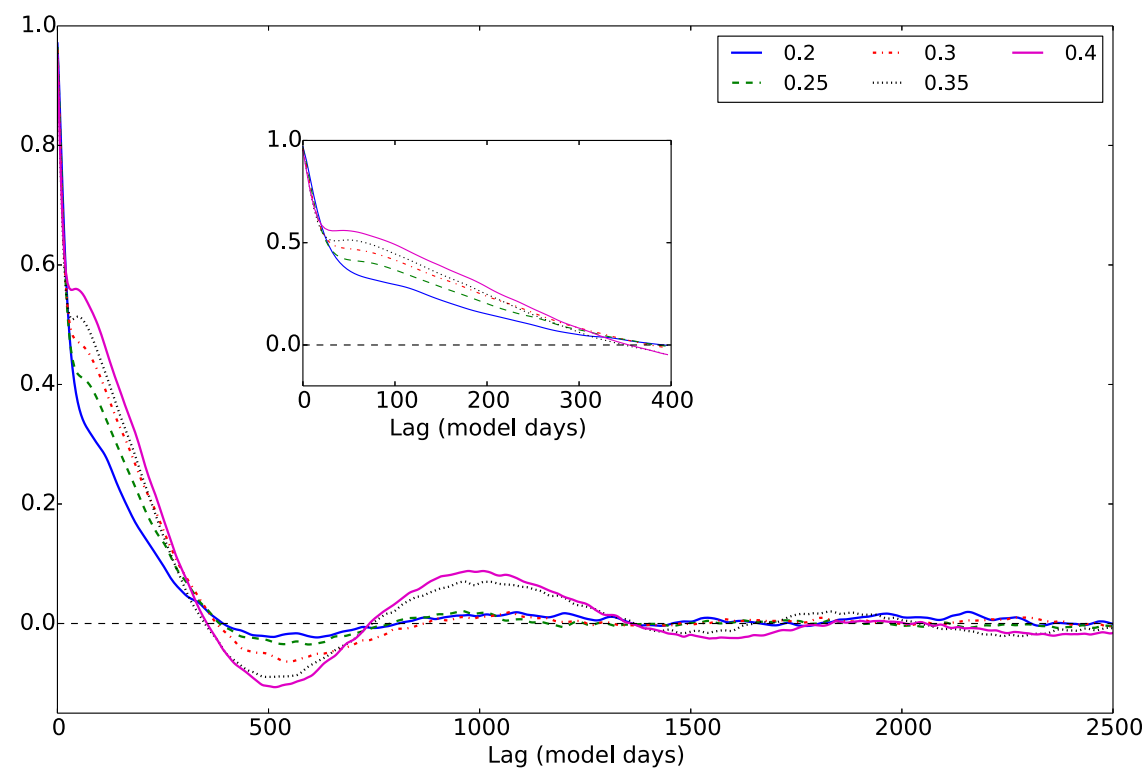

FIG. 5. Autocorrelation of the first principal component as $\beta$ is increased ( 5 model days $\approx 1$ Earth day). 

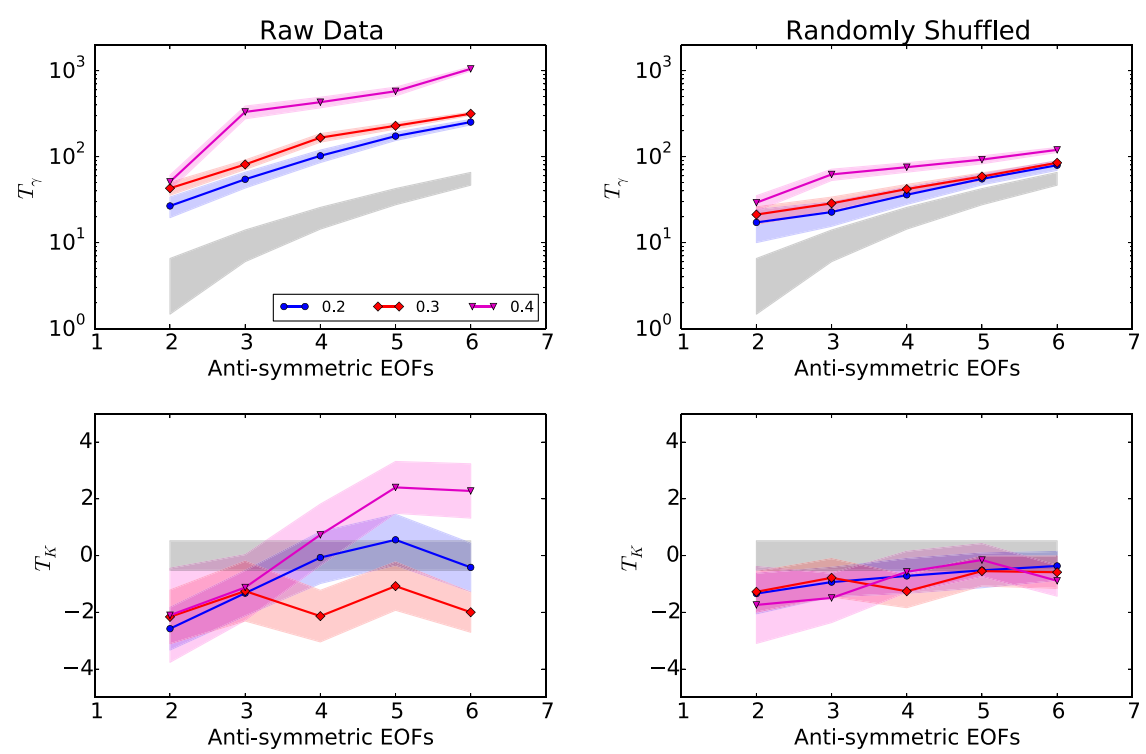

FIG. 6. (left) Ensemble-mean estimates of the multivariate (top) skewness $T_{\gamma}$ and (bottom) kurtosis $T_{K}$ test statistics as a function of the number of included antisymmetric EOFs for different values of $\beta$. The gray shaded regions show the $95 \%$ confidence intervals corresponding to a 10-member-mean sample multivariate skewness and kurtosis, when the underlying data are Gaussian and these statistics are distributed as explained in section $2 \mathrm{~d}$. The colored shading shows plus or minus one standard deviation divided by $\sqrt{10}$ of the estimates. (right) As in (left), but where the principal component time series are randomly disordered to remove the coupling between EOFs.

involves other aspects of the model besides the annular modes (which have little structure in the wings in this parameter setting). The gray lines in Figs. 2c,e mark the maximum of the model responses; a criterion that we have used to evaluate the performance of the FDT is the estimate of the response at these locations, which we term "max $\Delta u$." This criterion is relevant if one is more interested in capturing the magnitude of the responses in the center of the domain than in the wings.

As $\beta$ is increased, the responses become stronger and narrower. Focusing on the barotropic perturbation, we find a clear change in strength, shown by the black lines in Figs. 7a,c, which plot $\max \Delta u$ in each layer versus $\beta$ (note the near linear relationship between the two variables). More detail concerning the structure of the responses is given by the black lines of Fig. 8, which show that besides the change in strength, the response is narrower in the center and stronger in the wings as $\beta$ is increased. The baroclinic responses will be discussed in Fig. 16 and are similarly affected by increasing $\beta$.

These variations are partly the result of the changing role of eddies in determining the model's response. To demonstrate this, we have repeated the experiments with the nonlinear terms in the model removed, eliminating the eddy fluxes so that the balance in the model is between the forcing, the Newtonian relaxation, and the friction. Figure 9 compares the resulting "no eddy" responses to the two perturbations with the full model responses in the $\beta=0.2$ and $\beta=0.4$ cases (the no-eddy responses are independent of $\beta$ ). For the barotropic perturbation the $\beta=0.2$ case is actually quite similar to the no-eddy response, with the eddies simply acting to amplify and broaden the response in the center of the domain slightly as well as to add structure in the wings. This resemblance may be related to the similarity between the time-mean jet and the radiative equilibrium profile in this setting. However, as $\beta$ is increased the response of the full model changes (red lines in Fig. 9) while the no-eddy response is unaffected, showing that eddies increasingly push the full model's response away from the no-eddy response. In the baroclinic case there is no response in the lower layer when eddies are turned off, so that no matter what value $\beta$ takes, eddies play a large role in determining the response of the model. The FDT will have to capture this changing eddy behavior.

\section{FDT calculations}

\section{a. Choosing how many EOFs to retain}

Making an appropriate choice of how many degrees of freedom to retain is crucial for producing accurate FDT estimates. Given that we have already generated the model responses an obvious choice would be to keep 

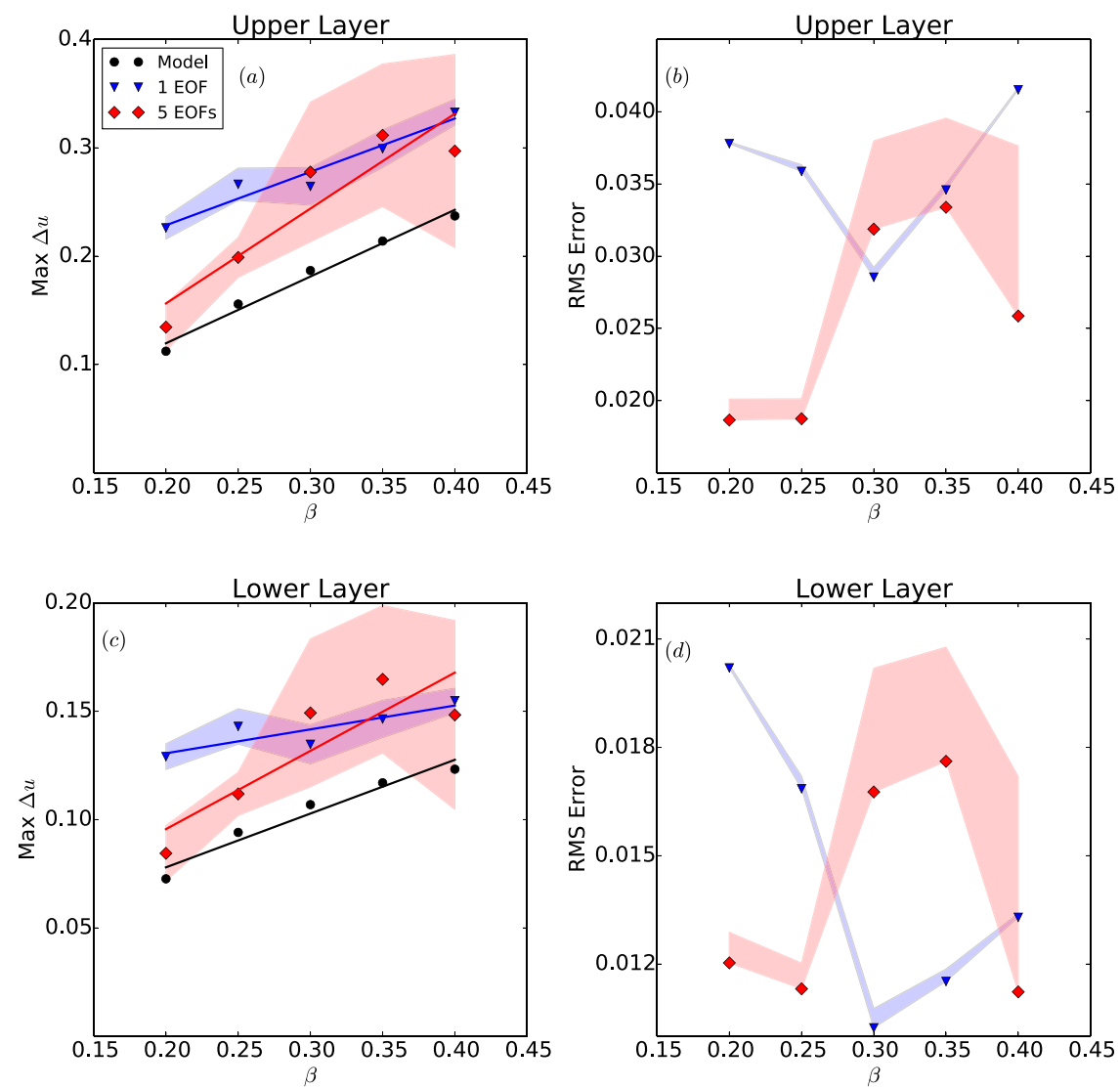

FIG. 7. (a) Variation of $\max \Delta u$ as $\beta$ is increased (black circles) and the corresponding ensemble-mean FDT estimates for the upper layer using the leading EOF (blue triangles) and the first five antisymmetric EOFs (red diamonds). The shading is the plus or minus one standard deviation envelope while the straight lines represent linear least squares fits to the data. (b) As in (a), but for the RMS error instead of $\max \Delta u$. (c),(d) As in (a),(b), but for the lower layer.

only those EOFs onto which these responses project; however, we would like to know how many EOFs to retain a priori, without resorting to running the model first; this is part of the motivation for using the FDT in the first place.

As can be seen from Fig. 10, the leading EOF explains between $65 \%$ and $75 \%$ of the variance for every value of $\beta$, quantifying the dominance of the annular mode in this model and suggesting that the most obvious strategy would be to retain only the leading EOF in each case. This is also appealing owing to the hypothesized connection between the response of the midlatitudes to perturbations and the autocorrelation time scale of the annular modes, and so this is the first strategy we have adopted. However, given that the responses have significant structure in the wings, it is likely that more EOFs are required for accurate FDT calculations. There are then two a priori criteria that could be used to decide how many EOFs to retain: 1) determine how many EOFs are well resolved and discard the rest or 2) retain the minimum number of EOFs required to explain some threshold of variance. Note that these are likely to be related: EOFs that explain significant variance will be excited more often and hence be better resolved. However, the choice of threshold is subjective and so we have focused on the first criterion.

To determine how many EOFs were well resolved we followed DelSole (1996) and calculated how many EOFs were well separated by the North et al. (1982) criterion. Here, well separated means that the separation between the eigenvalues corresponding to the EOFs is greater than the sampling error in the eigenvalues themselves. More explicitly, we calculated the value of $S_{n}$ for each eigenvalue, $\lambda_{n}$ :

$$
S_{n}=\frac{2 \lambda_{n}}{\lambda_{n-1}-\lambda_{n+1}}
$$

From Fig. 5 the decorrelation time is of order 1000 model days and each ensemble member contained $10^{5}$ 

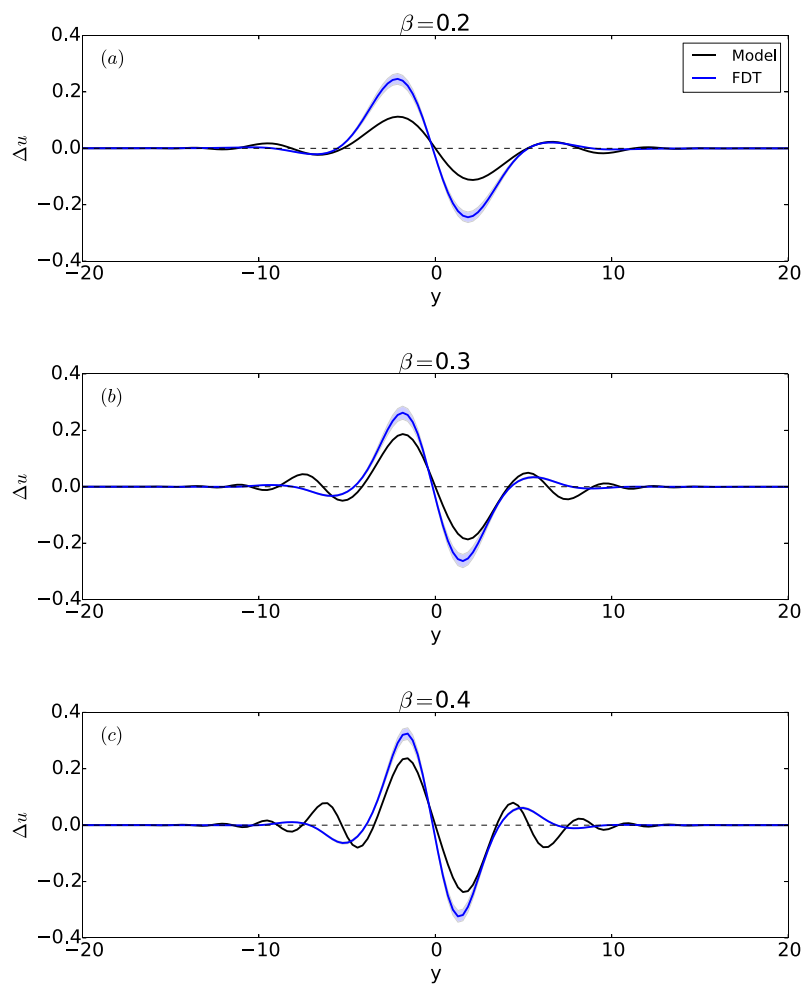

FIG. 8. Comparison of upper-layer model responses to the barotropic perturbation for three values of $\beta$ (black lines) and ensemblemean FDT estimates using the leading coupled EOF (blue lines) in each case. The shading is plus or minus one standard deviation.

model days so the relative error, $\varepsilon$, was of order $10 \%$. We used this to define an antisymmetric, coupled EOF as well separated when $\varepsilon S_{n}<1$ and the vertical black lines in Fig. 10 mark for each value of $\beta$ the first EOF that was not well separated by this criterion. In every case only the first five EOFs were well separated. The horizontal black lines in Fig. 10 also show that the first five EOFs explained roughly $95 \%$ of the variance in every case. For comparison, previous FDT studies have typically retained between $90 \%$ and $99 \%$ of the variance (Gritsun and Branstator 2007; Ring and Plumb 2008; Fuchs et al. 2015).

This is a plausible criteria, which can be used without considering the model responses, but there is a problem when applying this criterion to the case of a baroclinic perturbation. Returning to Fig. 2, the baroclinic forcing perturbation produces an equivalent barotropic response. This response projects strongly onto the leading EOF but the perturbation does not and instead projects strongly onto the sixth EOF. Examining the structure of the EOFs shows that for every value of $\beta$ this corresponds to an "equivalent" baroclinic EOF (layer values have opposite sign but different magnitude; see red line in Fig. 3b), in contrast to the first five EOFs, which are equivalent barotropic. Hence, it is unsurprising that this EOF was poorly resolved (note that it is not as smooth and antisymmetric as the leading EOF) and does not explain much variance, as the equivalent baroclinic variability of the two-layer model is overwhelmed by the equivalent barotropic variability. In every case the sixth EOF also decorrelates very quickly, typically in one or two model days, further suggesting that it is not being resolved from the noise and so will cause problems for the FDT.

Given the evident sampling error, we clearly would not wish to include the sixth EOF in our calculations; however, it appeared to be necessary for the baroclinic case. As such, for the barotropic case we only retained the first five EOFs while in the baroclinic case we performed the calculations using both five and six EOFs.

\section{b. Barotropic perturbation}

\section{1) ONE EOF}

We begin the presentation of our results by discussing how well the model responses to the barotropic perturbation were estimated using the leading EOFs. As has been mentioned, one of the motivations for this study was the hypothesis that the autocorrelation time scale of the annular modes controls the strength of the response of the midlatitudes to small perturbations. In the context of the FDT, this amounts to saying that the integral of the annular-mode autocorrelation can give an accurate estimate of the model's response.

To test this, the blue lines in Fig. 8 compare the ensemblemean FDT estimates of the upper-layer responses using the leading EOFs to the true responses for three different values of $\beta$. In each case 2000 model time units, or 400 Earth days, were used as the upper-limit $T$. This upper limit was chosen as the autocorrelation of the principal components had approximately decayed to zero in all cases at this lag (Fig. 5). The FDT overestimated the model's response and failed to capture the response in the wings; however, the estimates were still reasonable, particularly in the $\beta=0.3$ case, which had a very small root-mean-square (RMS) error (see Figs. $7 b, d$ ). The FDT was also able to at least approximate the difference in magnitudes between the responses of the two layers (Fig. 7).

Although a single EOF cannot be expected to capture the full structure of the response, an interesting question is whether it can capture how the strength of the response varies with $\beta$. To test this, we examined how the estimates of $\max \Delta u$ varied as $\beta$ was increased (Fig. 7). As was mentioned previously, there is a near linear relationship in both layers between $\max \Delta u$ and $\beta$, with a least squares fit to the data giving a linear slope of 0.61 in the upper layer and 0.25 in the lower layer. Repeating 

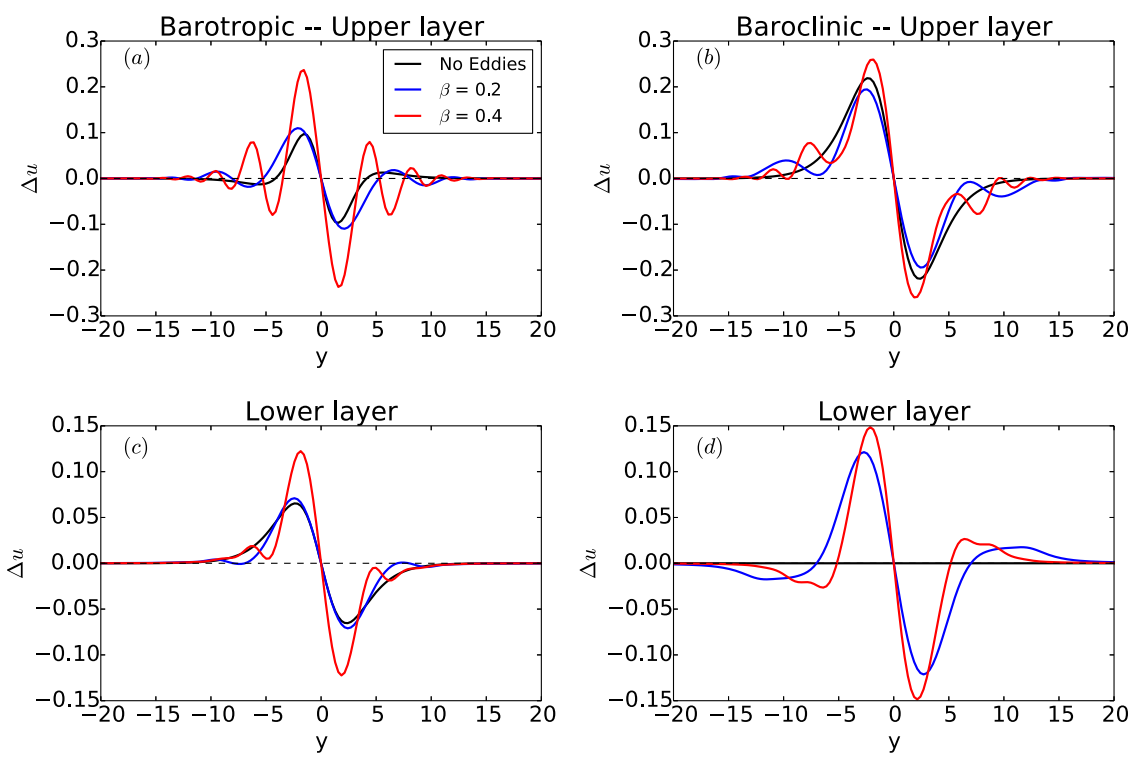

FIG. 9. (a),(c) The model response to the barotropic perturbation without eddies (black) and the model responses with eddies, and $\beta=0.2$ (blue) and $\beta=0.4$ (red). (b),(d) As in (a),(c), but for the baroclinic perturbation.

this calculation for the FDT estimates, slopes of 0.49 and 0.11 were obtained. These differ significantly from the true slopes but, given that the slopes are of the right sign and magnitude, the FDT using only the dominant EOF can be said to capture the qualitative changes in the linear response as supercriticality is varied.

To first order then, the FDT did a reasonable job of estimating the model's response with just the leading EOFs. To ensure that these estimates were robust to the choice of the upper limit of integration, $T$ was varied from 40 to 3000 model days in each case. From Fig. 5 one would expect the $\beta=0.4$ case to be the most sensitive to $T$ and so we show how the FDT estimate of $\max \Delta u$ in the upper layer was affected by changing $T$ in this setup in Fig. 11a (the results in the lower layer and for other values of $\beta$ were similar). The estimate was relatively stable for $T$ greater than 1000 days, though there was some oscillatory behavior owing to the structure at long lags. It is possible that better estimates could have been obtained in each case by choosing $T$ more carefully but we were not seeking to tune our calculations in this way and so felt comfortable using an upper limit of 2000 model days. Note that this is much longer than previous FDT studies, which have used values of $T$ closer to 30 Earth days ( 150 model days) and is necessary because of the unrealistically long decorrelation times in our model.

\section{2) FIVE EOFs}

The results of the previous section are promising; however, based on section 4a, five EOFs were expected to produce the best estimates. Hence, we repeated the calculations of the previous section with the first five EOFs, the results of which are shown for the upper layer in Fig. 12. A significantly better fit was now obtained, particularly in the wings, and though the FDT continued
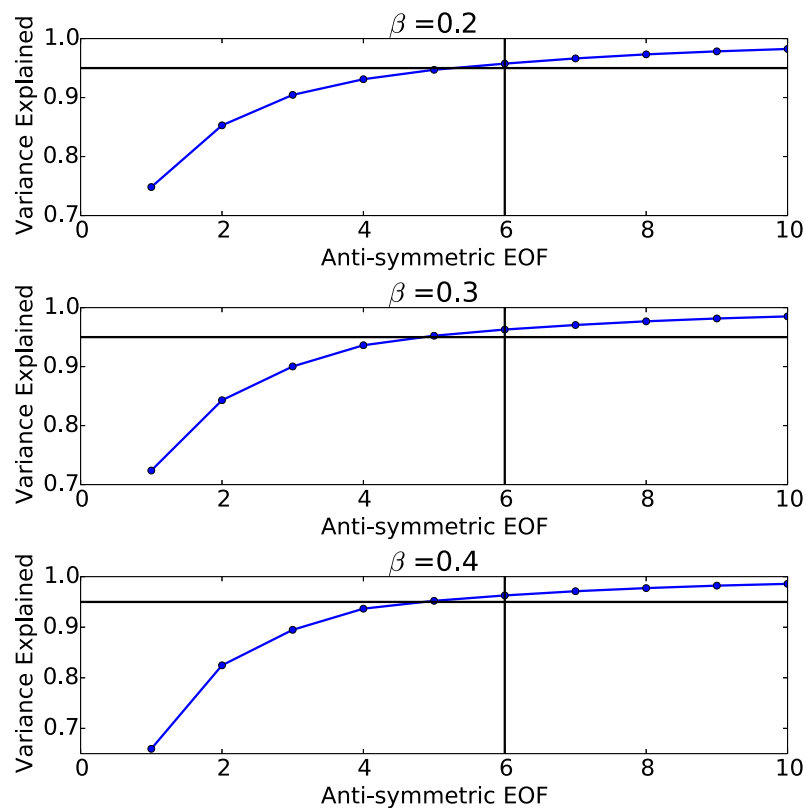

FIG. 10. The cumulative variance explained by the antisymmetric EOFs for three values of $\beta$. The horizontal black lines mark the $95 \%$ line while the vertical black lines denote the first EOF, which was not well separated. 

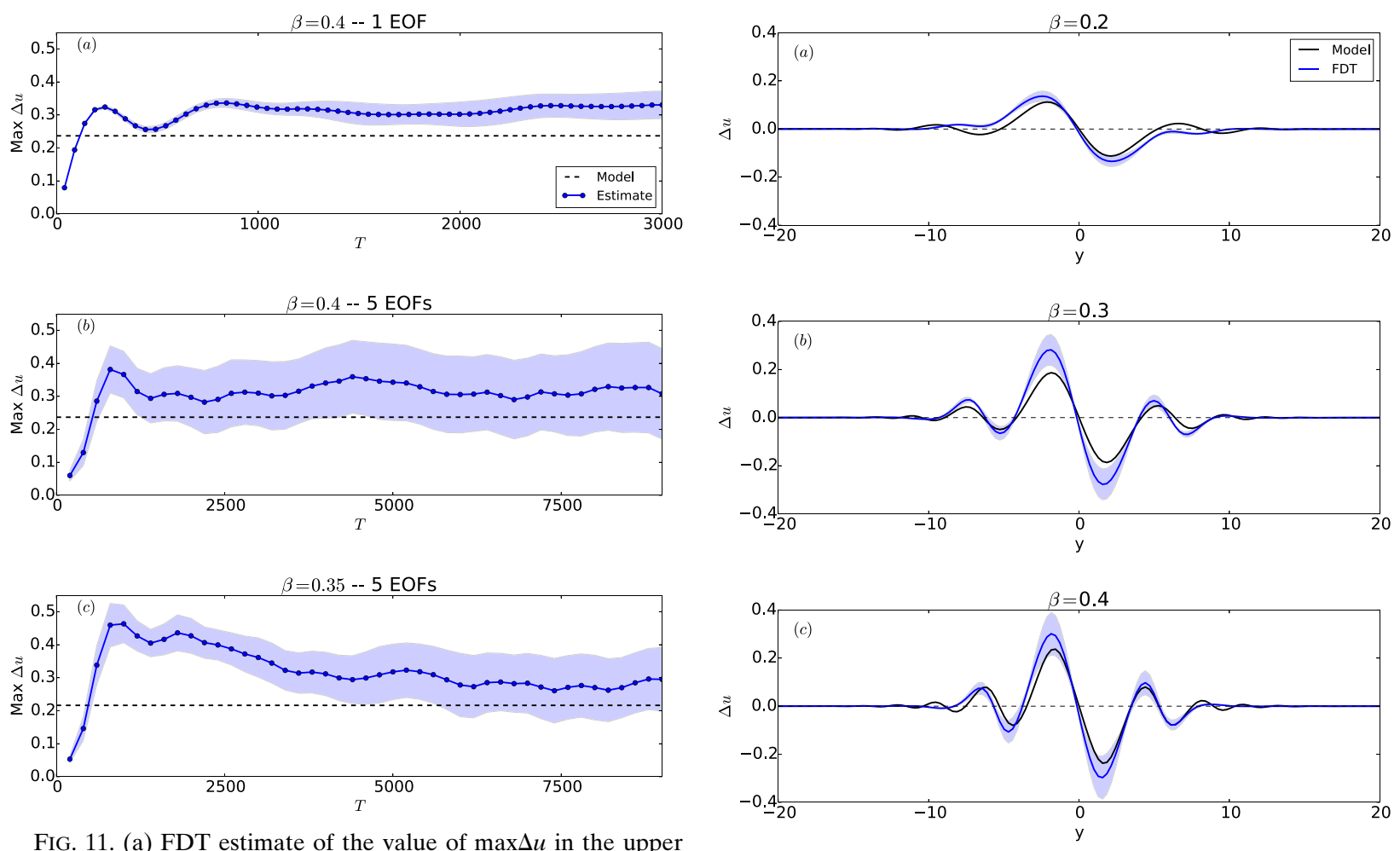

FIG. 11. (a) FDT estimate of the value of $\max \Delta u$ in the upper layer using the leading coupled EOF for the case $\beta=0.4$ as a function of the upper-integration limit $T$. The blue dots show the ensemble mean while the shading is plus or minus one standard deviation. The horizontal dashed line shows the value of $\max \Delta u$ for the model response. (b) As in (a), but using the first five antisymmetric coupled EOFs in the FDT calculations and (c) for the $\beta=0.35$ case also using five EOFs.

to overestimate the magnitude of the response, this overestimation was generally less than when only the leading EOFs were included. Figures $7 \mathrm{~b}, \mathrm{~d}$ quantify this by comparing the RMS error of the estimates using the leading EOF only and using the first five EOFs. In general, using five EOFs produced lower RMS errors, except for the $\beta=0.3$ and $\beta=0.35$ cases when the estimates using five EOFs overestimated the magnitude of the response more than the estimates using the leading EOF. However, even in these cases using five EOFs resulted in much better estimates of the wings of the responses. The cause of the overestimation is unclear but is presumably related to the non-Gaussian behavior mentioned in section 3. For instance, the overestimation and the sampling error generally increase with $\beta$ (Fig. 12), similar to how the data become less Gaussian.

As another way of presenting these results, in the left panels of Fig. 13 the upper-layer no-eddy response has been subtracted from every curve in Figs. 12a,c,e, leaving the "eddy only" response (the lower-layer results are similar). The ability of the FDT to capture the effects of eddies on the response is clearly quite good as the

FIG. 12. As in Fig. 8, but the FDT estimates used the first five antisymmetric coupled EOFs.

FDT was able to produce accurate estimates for the shape of the eddy-only response, though once again the response in the center of the domain was overestimated, particularly for larger values of $\beta$. Similarly, the right panels show the model response of the upper-layer potential vorticity flux and the corresponding FDT estimates (the potential vorticity flux response can be calculated directly from the estimates of the zonal-mean zonal wind response). These calculations show that the FDT is qualitatively capturing how the eddy responses vary with $\beta$.

Our a priori calculations suggested that five EOFs would be required to produce the best estimates but to check this we varied the number of EOFs included in our calculations from 1 to 15 . When fewer than five EOFs were used the results were not as accurate (sometimes the FDT estimates even had the wrong sign), suggesting that too-few degrees of freedom were included, whereas when six or more EOFs were included the results varied widely because of the sampling error. In line with our expectations then, five EOFs consistently produced the best FDT estimates in terms of the shapes and amplitudes of the responses. However, using five EOFs did not improve the estimate of the slope of $\max \Delta u$ versus $\beta$, giving slopes of 0.87 and 0.36 for the upper and lower layers, respectively (Fig. 7; note the larger sampling errors). These results also point to the importance of only keeping the 

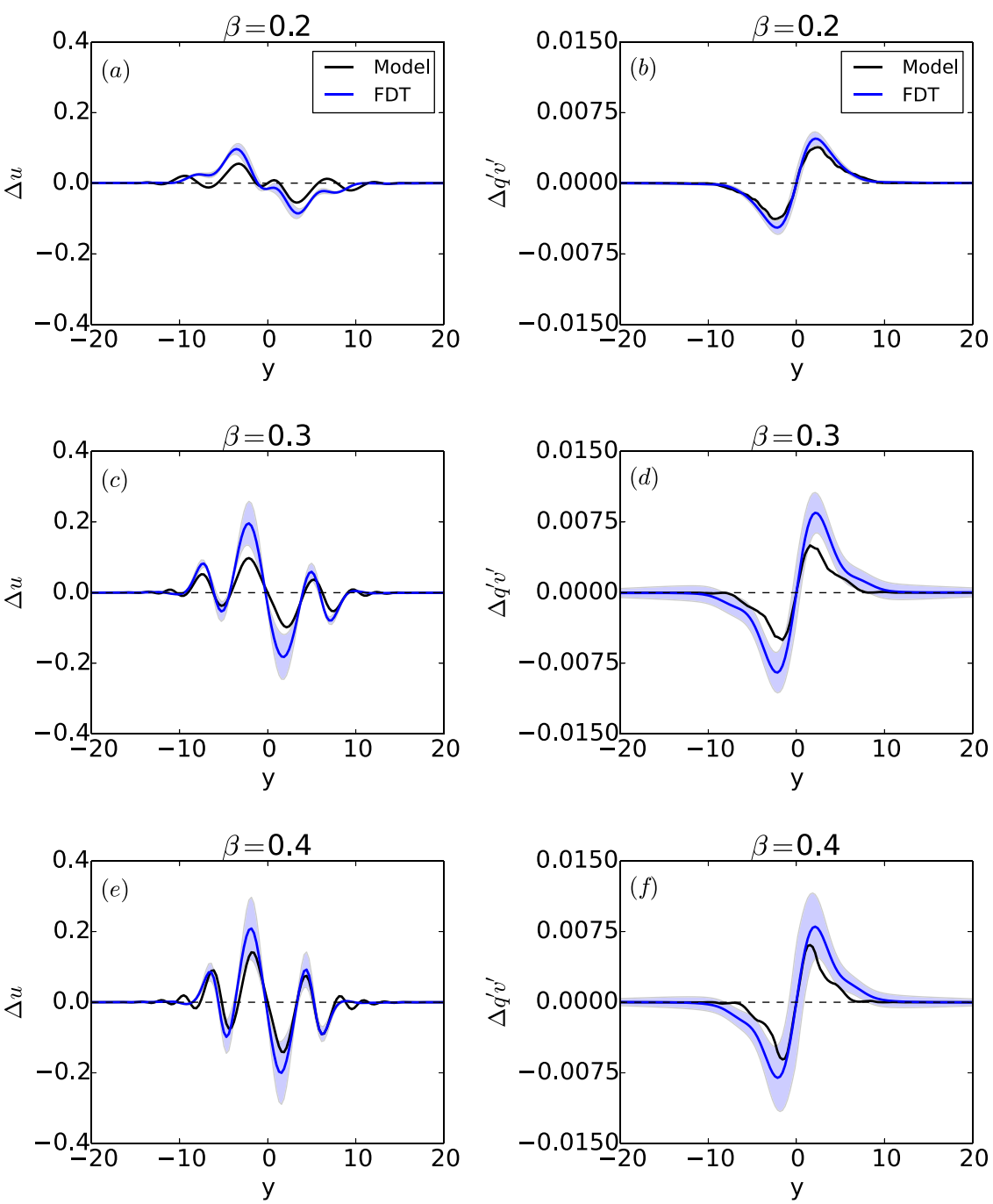

FIG. 13. (a),(c),(e) As in Fig. 12, but the no-eddy response has been subtracted from every curve. (b),(d),(f) The model response of the upper-layer potential vorticity flux in black and the corresponding FDT estimates in blue.

well-resolved EOFs. Choosing to retain $90 \%$ of the variance would have produced worse estimates and similarly if $99 \%$ of the variance had been retained, yet a priori there was no way of knowing that retaining $95 \%$ would work better than either of these.

To obtain these estimates a value of 2000 model days was again used for $T$ in all cases except when $\beta$ was set to 0.35 . In this case it was found that the estimates took much longer to converge (see Fig. 11a) and so the FDT estimate for $\beta=0.35$ case shown here was obtained using 5000 model days for $T$; in the other cases we continued using 2000 model days in order to limit the sampling error. It is unclear why this case took so much longer to converge as in the other cases the estimates had essentially converged after roughly 1500 model days (e.g., Fig. 11b), though it is noticeable that the FDT estimate for the $\beta=0.35$ case overshot significantly at short lags.

\section{c. Baroclinic perturbation}

The discussion in section 4a showed that the FDT was unlikely to produce a good estimate for the model's response to the baroclinic perturbation using this basis as the perturbation does not project onto the well-resolved EOFs. For instance, the left two panels of Fig. 14 show that when five EOFs were used in the $\beta=0.2$ case the FDT estimate substantially underestimated the response and the estimate was also of the wrong sign. The right panels then show that when six EOFs were used the FDT estimate had approximately the right magnitude but again had the wrong sign. The FDT performed just as poorly for other values of $\beta$. 

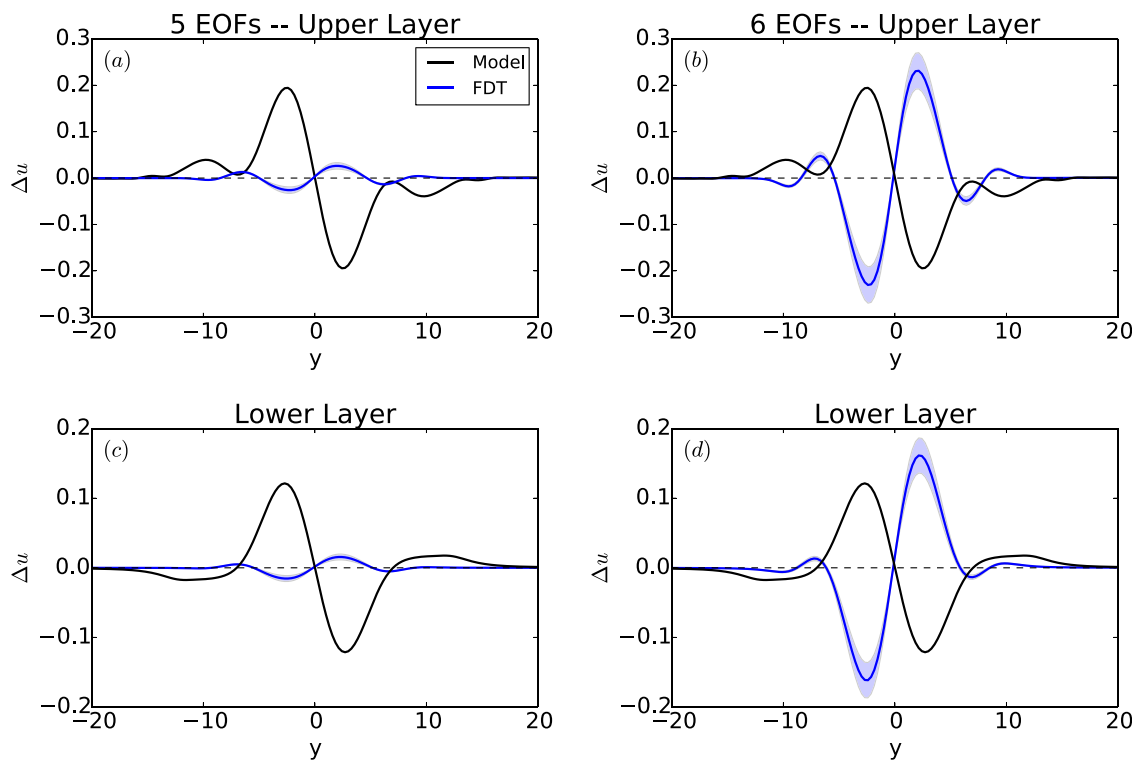

FIG. 14. Ensemble-mean FDT estimates for the model's response to the baroclinic perturbation in the case $\beta=0.2$. (a),(c) The results using the first five coupled antisymmetric EOFs and (b),(d) the results using the first six coupled antisymmetric EOFs. The color scheme is as in Fig. 8. (top) The upper layer and (bottom) the lower layer.

To get around the problem of capturing the perturbation while ensuring that the EOFs were well sampled, we repeated these calculations using two alternative bases. The first basis was constructed by separating the data into their barotropic and baroclinic components and then calculating a new set of EOFs. The barotropic variability is less dominant than the equivalent barotropic variability and so might not be as well sampled, but conversely the purely baroclinic variability might be easier to resolve than the equivalent baroclinic variability. Indeed, using the symmetry/asymmetry of the EOFs as a measure of the sampling errors, the baroclinic EOFs were better sampled. Only the leading barotropic and baroclinic EOFs were included in the FDT calculations (calculations in which more EOFs were included produced worse estimates); however, this was enough to ensure that both sets of perturbations and responses projected strongly onto the EOFs.

In the barotropic case these new EOFs performed worse than the coupled EOFs, as the resulting FDT estimates predicted that the model's response would decrease in strength with $\beta$. Nevertheless, the estimates were still reasonable in all cases apart from for $\beta=0.4$ (see Fig. 15). On the other hand, much better estimates for the baroclinic perturbation were obtained (Fig. 16; the agreement for the lower layer was similar) and the FDT now correctly predicted that the response would increase with $\beta$, though the slope was significantly overestimated: the response when $\beta=0.2$ was substantially underestimated while the response when $\beta=0.4$ was slightly overestimated. In all cases an upper limit of 2000 model days was again used and the estimates were checked to ensure that they were robust to the choice of $T$.

The FDT can thus produce reasonable estimates for the baroclinic case following the procedure from before but dividing up the data in such a way as to improve the sampling. We interpret this as saying that the sampling is the issue, not a more fundamental failure of the formalism due, in particular, to non-Gaussianity.

A second alternative basis was generated for each perturbation by first subtracting the variability associated with the forcing vector from the data and calculating EOFs for the remaining variability. These EOFs were then combined with the forcing vector to produce the new basis. New bases thus had to be tailored to each perturbation and could not be reused for other perturbations to the forcing. Also, the different forcing vectors were not independent of the EOFs and so the $\mathbf{C}(0)$ matrices were no longer diagonal. On the other hand, this choice of basis still does not assume anything about the form of the response.

Once these bases were generated only the well-separated EOFs were retained-in addition to the forcing vector-in order to reduce the sampling errors (only the leading EOF was well separated). This approach was very sensitive to the length of the dataset, so using the whole time series produced better, and more robust, estimates than taking the average of the ensemble members. However, once the estimates had converged they were quite accurate. The barotropic estimates were very similar to those using 

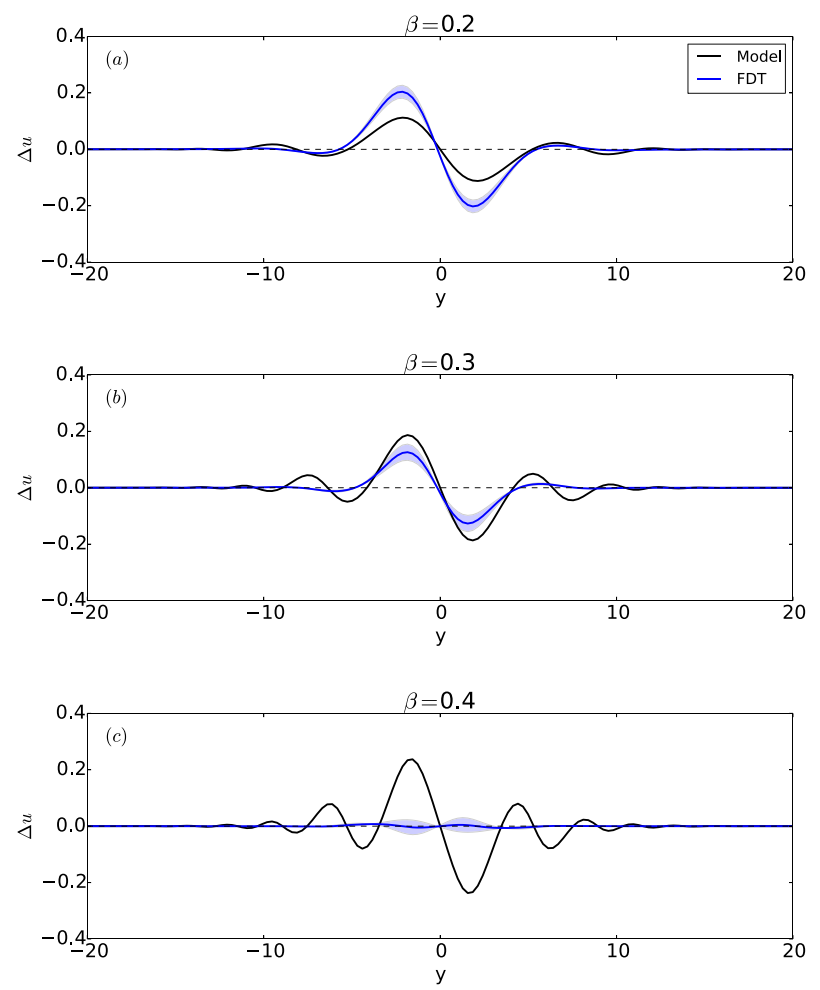

FIG. 15. Ensemble-mean FDT estimates for the upper-layer model responses to the barotropic perturbation using the leading barotropic and baroclinic EOFs for three values of $\beta$. The color scheme is as in Fig. 8.

the coupled EOFs, while the baroclinic estimates captured the leading order behavior for every case except when $\beta=0.2$ (Fig. 17).

\section{Conclusions}

In this work, we have used the FDT to estimate the response of a baroclinically unstable jet in a two-layer QG model on a $\beta$ plane in a periodic channel. The control climate that is perturbed is symmetric about the center of the channel. We consider two perturbations, both of which produce zonal wind responses that are antisymmetric about the center of the channel and are therefore annular-mode-like, but differ in the vertical structure of the forcing. For the barotropic perturbation the FDT gives robust and quantitatively useful estimates for a range of values of $\beta$ using the first five antisymmetric, coupled EOFs, though it consistently overestimates the response, perhaps owing to significant departures from Gaussianity in the data. This is exactly how many EOFs are well separated according to the criterion of North et al. (1982), though more rigorous testing would be required to check that this is a general result. Reasonable estimates are also obtained using just the leading EOF in each case and
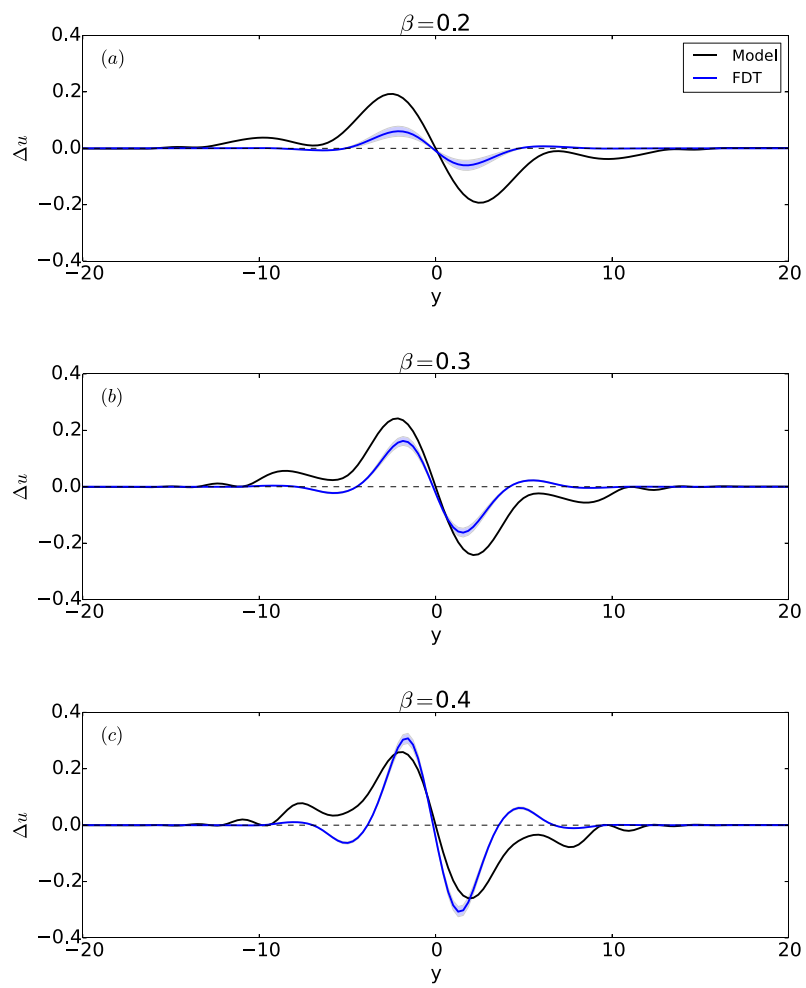

FIG. 16. As in Fig. 15, but for the baroclinic perturbation.

the FDT was able to approximately predict how the amplitude of the response increases with $\beta$. This adds to the evidence that the annular-mode time scale plays an important role in determining the response of the midlatitudes to small perturbations, though it should be noted that our results are still more qualitatively accurate than quantitative and it is not clear that inaccuracies in Ring and Plumb (2008) are a result of their use of a POP-based formalism.

Conversely, the baroclinic perturbation is more challenging for the FDT. The perturbation to the forcing projects too weakly onto the well-resolved EOFs for these to produce accurate estimates, but adding the poorly resolved EOFs that the forcing does project onto results in large errors. This agrees with the findings of Gritsun and Branstator (2007), who obtained worse FDT estimates for baroclinic forcings than for barotropic forcings. Our results suggest that the difficulty of predicting responses to forcings of this kind might be due to the fact that atmospheric variability is dominated by equivalent barotropic structures. To get around this problem we have devised two new strategies for performing the FDT calculations. In the first, the data were divided into their barotropic and baroclinic components and new EOFs were calculated. This alternative basis better resolves the baroclinic variability and so can handle the baroclinic perturbation. The second method uses bases tailored to each 

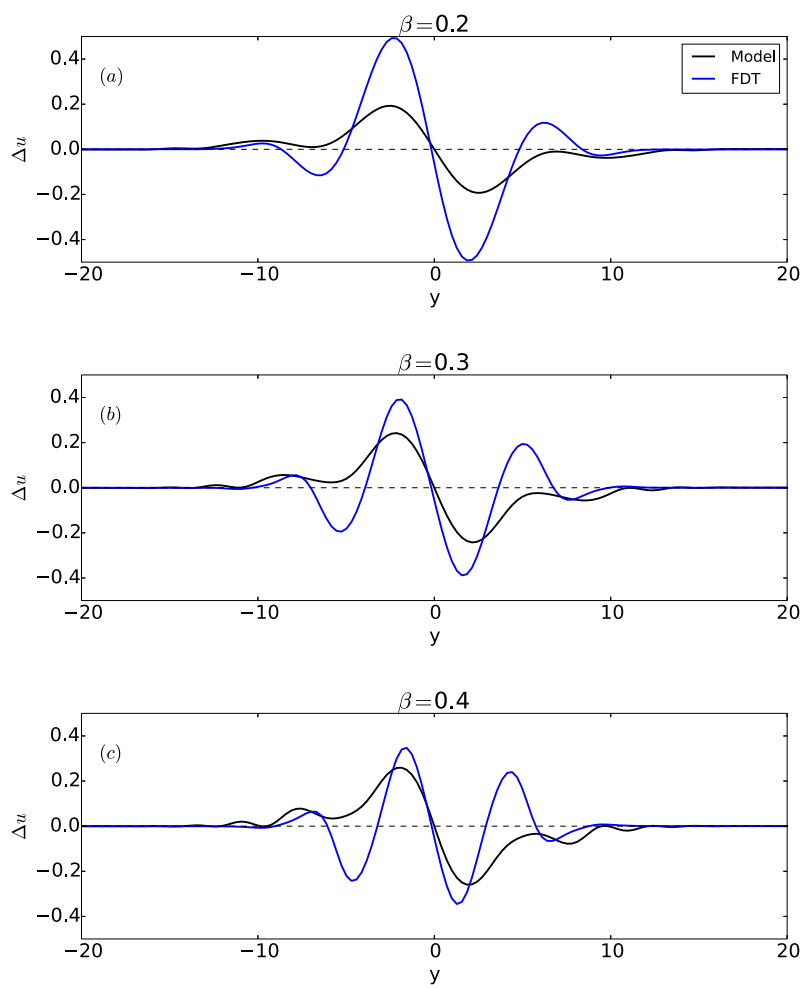

FIG. 17. As in Fig. 16, but the first basis vector took the form of the baroclinic perturbation. Only one EOF was retained in addition to this vector and these calculations used the whole time series rather than an ensemble (hence there are no estimates of the sampling error). The lower-layer results are similar.

forcing perturbation. This approach produced accurate estimates for both barotropic and baroclinic forcing perturbations but was more sensitive to the length of the time series.

Taken as a whole, these results show the importance of sampling when applying the FDT to climate systems. On the one hand, adding poorly resolved EOFs reduces the accuracy of FDT estimates, but the perturbation and the response must both be captured by the well-resolved EOFs in order to obtain accurate estimates. This is similar to the findings of Fuchs et al. (2015), who obtained better FDT estimates for the response of an intermediatecomplexity GCM to realistic heating perturbations than to idealized "hot spot" perturbations.

The strategy of using the perturbation to the forcing as the leading basis vector might provide a way around some of these difficulties. This approach is different from that used in previous studies in that it requires calculating a new basis for each forcing perturbation but still does not require knowledge of the model's response. Hence, future FDT studies might consider a hybrid strategy in which a conventional basis is used for forcing perturbations that project strongly onto the leading modes of variability, while more specialized bases are used for perturbations that are poorly captured by the conventional EOFs. As a topical example, it may be possible to use a carefully constructed basis to produce FDT estimates for the response in more complex models of the midlatitudes to high-latitude stratospheric cooling.

Finally, although our results have investigated the importance of resolution, there are still several other outstanding issues with using the FDT for climate purposes. For instance, we have suggested that the overestimates of the magnitude of the response are correlated with nonGaussian behavior in the model, but we have been unable to show quantitatively how departures from Gaussianity bias FDT estimates. Another question is how the importance of resolution varies as the size of the system increases. The system considered here has relatively few active degrees of freedom because of its zonal symmetry and because of the symmetry of the control climate about the center of the channel, and we work with five to six EOFs. In contrast, in the study of Gritsun and Branstator (2007) the linear operator involved 1800 three-dimensional EOFs. For such a large system it is possible that even if a few EOFs were poorly sampled, these would constitute a much smaller fraction of the total number of included degrees of freedom and so their inclusion would not affect the FDT estimates as significantly as including a single additional EOF can here. In any case, although progress is still needed before the FDT is ready to quantitatively address larger questions such as estimating climate responses from observations, we have shown here that it can in some cases reproduce the response of a two-layer model to small perturbations in forcing with considerable accuracy. A linear, perturbative theory can thus be substituted for the eddy flux theories otherwise needed to predict these responses.

Acknowledgments. It is a pleasure to thank Tim DelSole, Grant Branstator, Sonya Legg, and Steve Garner for helpful discussions and comments on earlier versions of this manuscript as well as three anonymous reviewers. We also thank Stephan Fueglistaler for making the unpublished manuscript of Cooper and Haynes available to us. NJL was funded by the NOAA/Princeton University Cooperative Institute for Climate Science.

\section{APPENDIX}

\section{Cooper-Haynes Algorithm}

To integrate the lag-covariance matrices we have used an algorithm proposed by Cooper and Haynes in an unpublished manuscript. First note that we are integrating 


$$
\int_{0}^{\infty} \mathbf{C}(\tau) d \tau \approx \int_{0}^{T} \mathbf{C}(\tau) d \tau
$$

For a particular lag $\tau$ the entry in $\mathbf{C}(\tau)$ for two elements, $a$ and $b$, of the model state vector $\mathbf{X}$ is

$$
\mathbf{C}(\tau)_{a b}=\frac{1}{n-q-1} \sum_{p=1}^{n-q} x_{p} y_{q+p}
$$

where $n$ is the total length of the time series, $q$ is the number of data points representing a lag $t$ and

$$
\begin{aligned}
& x_{a}=X_{a}(t)-\overline{X_{a}}, \\
& y_{b}=X_{b}(t)-\overline{X_{b}} .
\end{aligned}
$$

Now taking $r$ to be the number of data points representing a lag of $T$ the trapezoid rule can be used to write the integral as

$$
\int_{0}^{\infty} \mathbf{C}(\tau) d \tau \approx \frac{\Delta \tau}{2(n-1)} \sum_{p=1}^{n} x_{p} y_{p}+\sum_{q=1}^{r} \frac{\Delta \tau}{n-q-1} \sum_{p=1}^{n-q} x_{p} y_{q+p},
$$

where $\Delta \tau$ is the spacing in time of the data points. The last term on the right-hand side can be approximated as (for $r \ll n$ )

$$
\begin{aligned}
\sum_{q=1}^{r} \frac{\Delta \tau}{n-q-1} \sum_{p=1}^{n-q} x_{p} y_{q+p} & \approx \frac{\Delta \tau}{n-r-1} \sum_{q=1}^{r} \sum_{p=1}^{n-q} x_{p} y_{q+p}, \\
& =\frac{\Delta \tau}{n-r-1} \sum_{p=1}^{n-q} x_{p}\left(\sum_{q=1}^{r} y_{q+p}\right) .
\end{aligned}
$$

The sum in parentheses can then be updated from the $p-1$ th operation using just one subtraction and one addition operation. The algorithm thus takes of the order $n$ operations, so that the whole matrix $\mathbf{C}(\tau)$ can be integrated using $d^{2} n$ operations, where $d$ is the spatial dimension of the dataset.

\section{REFERENCES}

Abramov, R. V., and A. J. Majda, 2009: A new algorithm for lowfrequency climate response. J. Atmos. Sci., 66, 286-309, doi:10.1175/2008JAS2813.1.

Bell, T. L., 1980: Climate sensitivity from fluctuation-dissipation: Simple model tests. J. Atmos. Sci., 37, 1700-1707, doi:10.1175/ 1520-0469(1980)037<1700:CSFFDS >2.0.CO;2.
Butler, A. H., D. W. J. Thompson, and R. Heikes, 2010: The steadystate atmospheric circulation response to climate change-like thermal forcings in a simple general circulation model. J. Climate, 23, 3474-3474, doi:10.1175/2010JCLI3228.1.

Chan, C. J., and R. A. Plumb, 2009: The response of the troposphere to stratospheric perturbations and its dependence on the state of the troposphere. J. Atmos. Sci., 66, 2107-2115, doi:10.1175/2009JAS2937.1.

Cooper, F. C., and P. H. Haynes, 2011: Climate sensitivity via a nonparametric fluctuation-dissipation theorem. J. Atmos. Sci., 68, 937-953, doi:10.1175/2010JAS3633.1.

DelSole, T., 1996: Can quasigeostrophic turbulence be modeled stochastically? J. Atmos. Sci., 53, 1617-1633, doi:10.1175/ 1520-0469(1996)053<1617:CQTBMS > 2.0.CO;2.

Fuchs, D., S. Sherwood, and D. Hernandez, 2015: An exploration of multivariate fluctuation dissipation operators and their response to sea surface temperature perturbations. J. Atmos. Sci., 72, 472-486, doi:10.1175/JAS-D-14-0077.1.

Gerber, E. P., S. Voronin, and L. M. Polvani, 2008: Testing the annular mode autocorrelation time scale in simple atmospheric general circulation models. Mon. Wea. Rev., 136, 1523-1535, doi:10.1175/2007MWR2211.1.

Gritsun, A., and G. Branstator, 2007: Climate response using a threedimensional operator based on the fluctuation-dissipation theorem. J. Atmos. Sci., 64, 2558-2575, doi:10.1175/JAS3943.1.

Leith, C. E., 1975: Climate response and fluctuation dissipation. J. Atmos. Sci., 32, 2022-2026, doi:10.1175/1520-0469(1975)032<2022: CRAFD $>2.0 . \mathrm{CO} ; 2$.

Lorenz, D. J., and J. L. Hartmann, 2001: Eddy-zonal flow feedback in the Southern Hemisphere. J. Atmos. Sci., 58, 3312-3327, doi:10.1175/1520-0469(2001)058<3312:EZFFIT>2.0.CO;2.

Majda, A. J., R. Abramov, and M. Grote, 2005: Information Theory and Stochastics for Multiscale Nonlinear Systems. American Mathematical Society, $133 \mathrm{pp}$.

_ B. Gershgorin, and Y. Yuan, 2010: Low-frequency climate response and fluctuation-dissipation theorems: Theory and practice. J. Atmos. Sci., 67, 1186-1201, doi:10.1175/2009JAS3264.1.

Martynov, R. S., and Y. M. Nechepurenko, 2004: Finding the response matrix for a discrete linear stochastic dynamical system. Comput. Math. Math. Phys., 44, 771-781, doi:10.1134/ S0965542506070062.

North, G. R., T. L. Bell, R. F. Calahan, and F. J. Moeng, 1982: Sampling errors in the estimation of empirical orthogonal functions. Mon. Wea. Rev., 110, 699-706, doi:10.1175/ 1520-0493(1982)110<0699:SEITEO > 2.0.CO;2.

Ring, M. J., and R. A. Plumb, 2008: The response of a simplified GCM to axisymmetric forcings: Applicability of the fluctuation-dissipation theorem. J. Atmos. Sci., 65, 3880-3898, doi:10.1175/2008JAS2773.1.

Wilks, D. S., 2006: Statistical Methods in the Atmospheric Sciences. 2nd ed. International Geophysics Series, Vol. 91, Academic Press, 648 pp.

Zurita-Gotor, P., 2014: On the sensitivity of zonal index persistence to friction. J. Atmos. Sci., 71, 3788-3800, doi:10.1175/JAS-D-14-0067.1. J. Blanco-Fuentes, and E. P. Gerber, 2014: The impact of baroclinic eddy feedback on the persistence of jet variability in the two-layer model. J. Atmos. Sci., 71, 410-429, doi:10.1175/ JAS-D-13-0102.1. 